\title{
BENCH-SCALE DEMONSTRATION OF BIOLOGICAL PRODUCTION OF ETHANOL FROM COAL SYNTHESIS GAS
}

\author{
Quarterly Report \\ September 24, 1992 - December 31, 1992
}

Contractor: Engineering Resources, Inc.

Contract No.: DE-AC22-92PC92118

Contract Duration: September 24, 1992 - September 23, 1994

\section{SUMMARY}

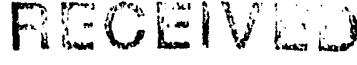

JUN 1419393

$0 S T I$

Culture isolation and selection studies are being performed in order to select the best biological system for bench-scale studies in producing ethanol from syngas components. Three isolates have been found which produce more than $2 \mathrm{~g} / \mathrm{L}$ ethanol from $\mathrm{CO}$ and $\mathrm{CO}_{2} / \mathrm{H}_{2}$ in batch culture. These low concentrations are actually quite promising since Clostridium ljungdahlii, strain PETC, performs well in continuous culture but produces only small concentrations of ethanol in batch culture after several weeks of incubation. Two of the isolates have been utilized in the CSTR, where 90 percent CO conversions have been noted, while producing up to $2 \mathrm{~g} / \mathrm{L}$ ethanol, in preliminary studies. CSTR studies will continue until steady state is reached.

\section{INTRODUCTION}

An anaerobic bacterium has been isolated from natural sources that converts the components of synthesis gas $\left(\mathrm{CO}, \mathrm{H}_{2}, \mathrm{CO}_{2}\right)$ into ethanol. This organism, the only one known at that time to produce ethanol from synthesis gas, has been identified as a new clostridial strain and has been named Clostridium ljungdahlii, strain PETC. Ethanol is produced according to the following stoichiometry:

$$
\begin{aligned}
& 6 \mathrm{CO}+3 \mathrm{H}_{2} \mathrm{O} \rightarrow \mathrm{CH}_{3} \mathrm{CH}_{2} \mathrm{OH}+4 \mathrm{CO}_{2} \\
& 2 \mathrm{CO}_{2}+6 \mathrm{H}_{2} \rightarrow \mathrm{CH}_{3} \mathrm{CH}_{2} \mathrm{OH}+3 \mathrm{H}_{2} \mathrm{O}
\end{aligned}
$$

Acetic acid is also produced as a by-product by the equations:

$$
\begin{aligned}
& 4 \mathrm{CO}+2 \mathrm{H}_{2} \mathrm{O} \rightarrow \mathrm{CH}_{3} \mathrm{COOH}+2 \mathrm{CO}_{2} \\
& 2 \mathrm{CO}_{2}+4 \mathrm{H}_{2} \rightarrow \mathrm{CH}_{3} \mathrm{COOH}+2 \mathrm{H}_{2} \mathrm{O}
\end{aligned}
$$

Therefore, all the components of synthesis gas can be utilized.

Initial studies with $C$. ljungdahlii, strain PETC, showed that under normal conditions, the organism produces primarily acetic acid (ETOH:HAC $=1: 20$ ). Subsequent studies with this organism have increased the ratio of ETOH:HAC to 3:1 by manipulating the $\mathrm{pH}$ and fermentation parameters. Later studies resulted in nearly eliminating acetate as a product by altering the growth medium and achieving high cell concentrations. A new isolate was recently discovered by Engineering Resources that produces primarily ethanol under normal conditions.

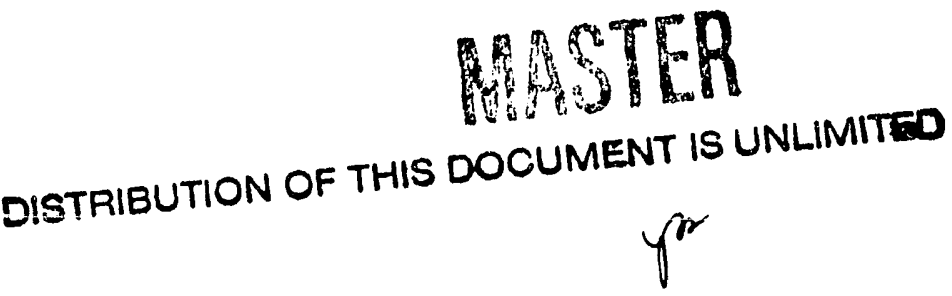


These studies, as well as studies to evaluate the performance at elevated pressure, are continuing. They have enabled the development of a conceptual design for a process to produce ethanol from coal synthesis gas. The most recent experimental results suggest that ethanol and acetate are produced in a series pathway. Therefore, the process must be designed to enable rapid removal of ethanol as it is produced to avoid conversion to acetate. The process is quite simple, consisting of a bioreactor and an ethanol recovery system. Synthesis gas would be sparged into the bioreactor, where a culture of $C$. ljungdahlii, strain PETC, or the new isolate, would be maintained. The $\mathrm{CO}, \mathrm{H}_{2}$ and $\mathrm{CO}_{2}$ would be converted into ethanol, which would be excracted from the aqueous culture medium into an organic solvent. The ethanol product would then be recovered by distillation and the solvent recycled. The cells would be retained in the bioreactor with a hollow fiber membrane, so that inhibition from contact with the solvent can be eliminated.

Preliminary economics indicate that the capital cost for the bioprocess to handle synthesis gases from a gasifier processing 1000 tons coal per day would be only $\$ 30$ million. The revenue generated from ethanol at current market prices is $\$ 66$ million per year, based on current yields of about 150 gallons per ton. These economics are quite promising and demonstrate that commercialization can be achieved in the near term. In order to reduce this technology to pracicice over the next four years, a two-year bench scale demonstration is necessary, followed by a two-year larger scale prototype demonstration.

The purpose of this two-year project is to develop and operate a bench scale unit for the production of ethanol from coal synthesis gas. This unit will include a bioreactor, cell recycle membrane, extraction unit and distillation column. Recycle loops for culture medium and solvent will be incorporated. Studies will be conducted to select the best ethanol culture, $C$. ljungdahlii, strain PETC, or the new isolate; and to identify the best solvent for ethanol extraction. Prolonged operation of the unit over a one year period will be conducted to demonstrate the viability of the culture and product recovery system and to identify potential problems with recycle loops. Scale-up parameters for the system will be developed for the design of the prototype and commercial units. The economics for a commercial facility will be projected to identify high cost areas for concentrated research.

\section{PURPOSE}

The purpose of this report is to present results from ongoing culture isolation and selection studies aimed at obtaining the best biological system for ethanol production from syngas. Several promising isolates have been obtained in addition to $C$. ljungdahlii, strain PETC, and are being utilized in batch and continuous culture comparison studies. Once the best culture has been selected, bench-scale studies will be initiated.

\section{RESEARCH ACTIVITY}

\section{Task 2. Culture Selection}

Several isolates have been obtained from natural sources that are capable of producing ethanol from $\mathrm{CO}$ and $\mathrm{CO}_{2} / \mathrm{H}_{2}$ in synthesis gas. An experimental program has been designed to evaluate these cultures in batch and continuous culture to select the best bacterium for benchscale operation. 


\section{Batch Studies}

Batch culture experiments have been performed with several enrichments that are able to produce ethanol from syngas. The cultures were grown anaerobically in mineral medium with 1.0 $\mathrm{g} / \mathrm{L}$ trypticase at $37^{\circ} \mathrm{C}$. The $\mathrm{pH}$ was maintained at a level below $\mathrm{pH} 5.0$ in order to facilitate ethanol production. Samples were taken periodically and analyzed for gas phase composition, liquid phase product (ethanol or acetate) concentrations and reaction $\mathrm{pH}$.

Three enrichments have been obtained which can produce more than $2 \mathrm{~g} / \mathrm{L}$ ethanol in batch culture. This batch ethanol concentration is considered to be quite good, since $C$. ljungdahlii, strain PETC, has performed poorly in batch culture, producing only small concentrations of ethanol after several weeks of incubation. Product formation by two of these isolates is shown in Figures 1 and 2. Isolate ERI2 produced nearly $1.5 \mathrm{~g} / \mathrm{L}$ ethanol in 11 days, with an equal concentration of acetic acid (see Figure 1). Isolate ERI8 produced $2.5 \mathrm{~g} / \mathrm{L}$ ethanol in 8 days, with only $1.0 \mathrm{~g} / \mathrm{L}$ acetic acid formed as a by-product (see Figure 2). Both isolates consumed both $\mathrm{CO}$ and $\mathrm{H}_{2}$ rapidly, requiring re-gassing of the batch bottle reactors at least once per day.

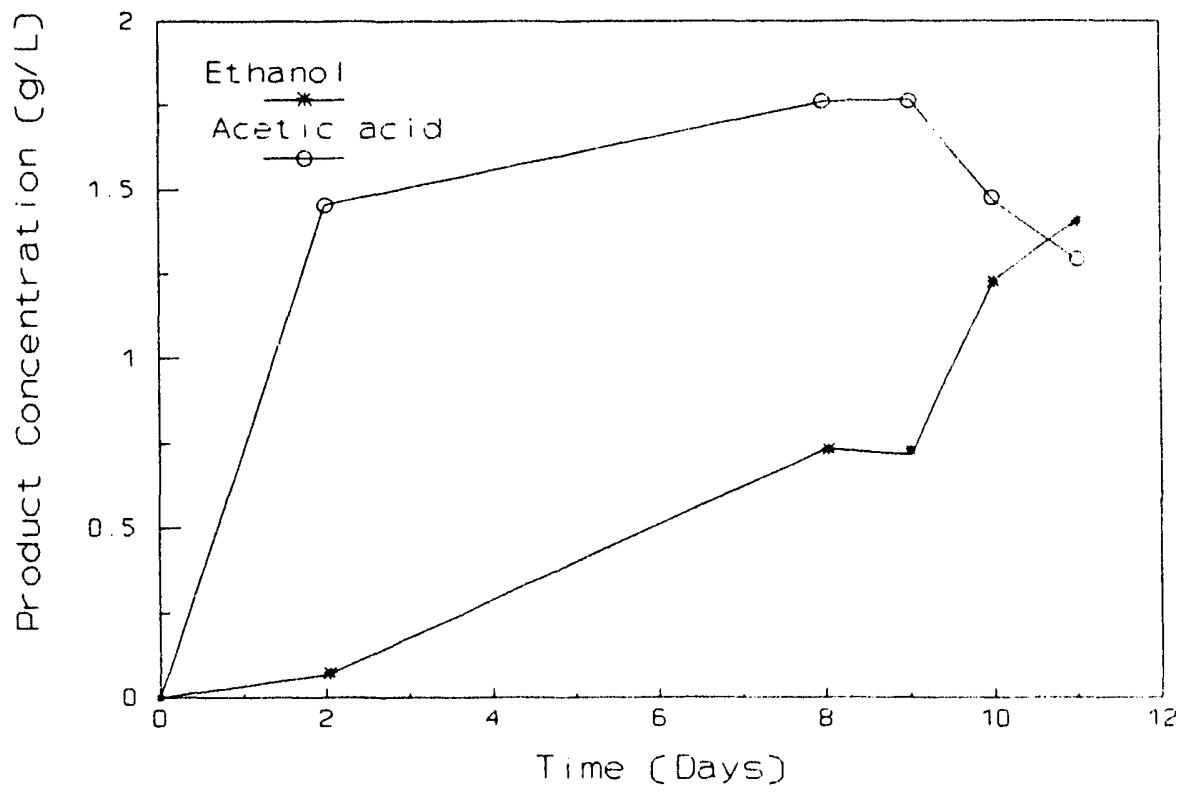

Figure 1. Ethanol and Acetic Acid Production from Synthesis Gas by Isolate ERI2 in Batch Culture

The production history in forming ethanol and acetate by the two isolates was quite different. Isolate ERI2 produced $1.7 \mathrm{~g} / \mathrm{L}$ acetate in 8 days while, at the same time, producing about $0.7 \mathrm{~g} / \mathrm{L}$ ethanol. The ethanol concentration then rapidly increased to $1.5 \mathrm{~g} / \mathrm{L}$ over the next 3 days, while the acetate concentration decreased to $1.3 \mathrm{~g} / \mathrm{L}$. By contrast, isolate ERI 8 produced $1.0 \mathrm{~g} / \mathrm{L}$ acetate after 2 days and held this concentration for the remaining 6 days of the experiment. The corresponding ethanol concentration gradually increased to $0.3 \mathrm{~g} / \mathrm{L}$ over the 


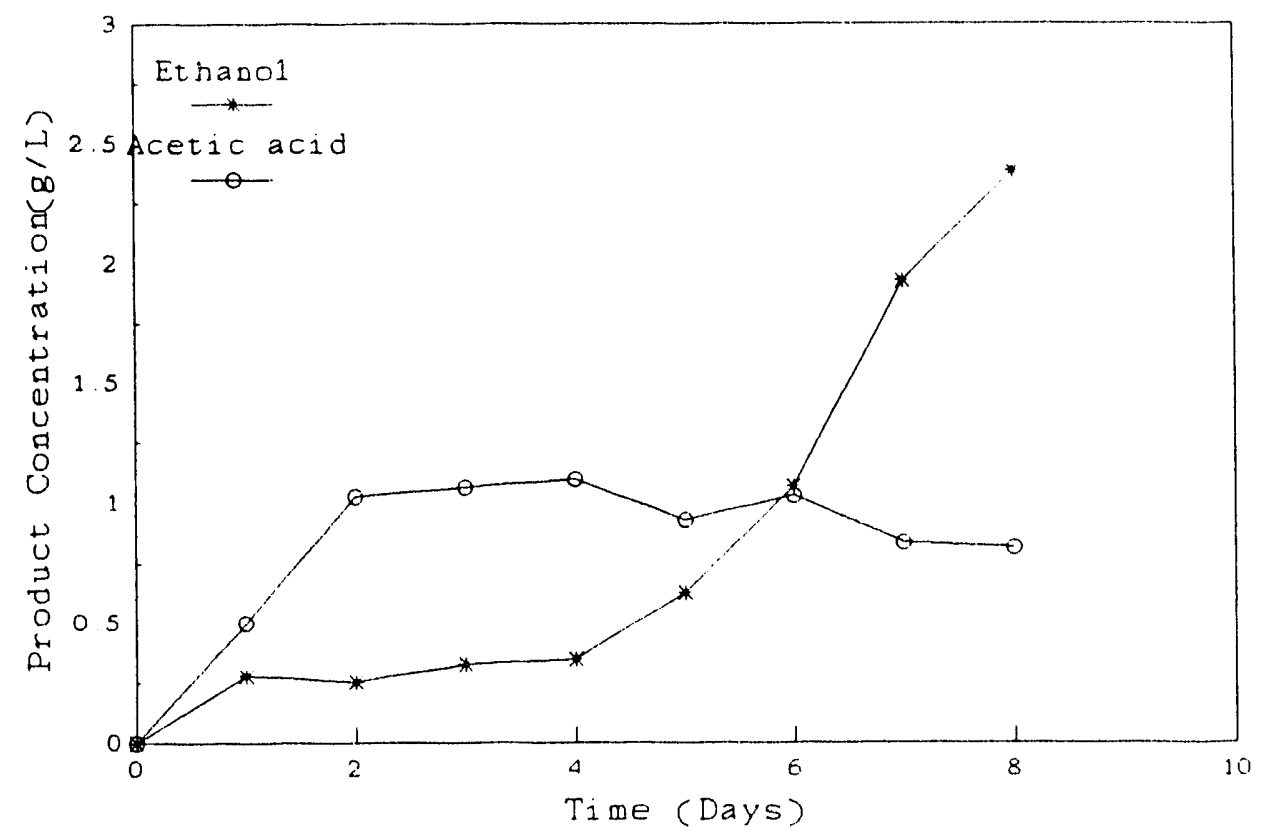

Figure 2. Ethanol and Acetic Acid Production from Synthesis Gas by Enrichment ERI8 in Batch Culture

first 4 days of the experiment before rapidly increasing to $2.5 \mathrm{~g} / \mathrm{L}$. C. ljungdahlii, strain PETC, behaves more like isolate ERI2, where the high acetate concentration reduces in favor of ethanol with time. These differences in ethanol and acetate formation rates will be studied further over the next month.

\section{CSTR Studies}

Because of the promising ethanol production data from batch culture studies, the two isolates were inoculated into CSTRs. Both cultures employed a mineral medium with $1 \mathrm{~g} / \mathrm{L}$ trypticase at $\mathrm{pH} 4.0$. The reactor temperature was $38.5-39.0^{\circ} \mathrm{C}$ and the agitation rate was 700 $\mathrm{rpm}$. Synthesis gas was introduced into the reactor at $5 \mathrm{~mL} / \mathrm{min}$ and liquid medium was fed at a dilution rate of $0.06 \mathrm{hr}^{-1}$.

The performance of isolate ERI2 in the CSTR for a short period of time is shown in Figures 3 and 4. As is noted in Figure 3, the CO conversion reached 90 percent and the $\mathrm{H}_{2}$ conversion reached $20-30$ percent in 4 days. The corresponding product concentrations were 2 $\mathrm{g} / \mathrm{L}$ ethanol and $4.3 \mathrm{~g} / \mathrm{L}$ acetate. These results are encouraging for this early stage of the fermentation. The performance of the culture will be monitored closely until steady state is reached.

\section{DISCLAIMER}




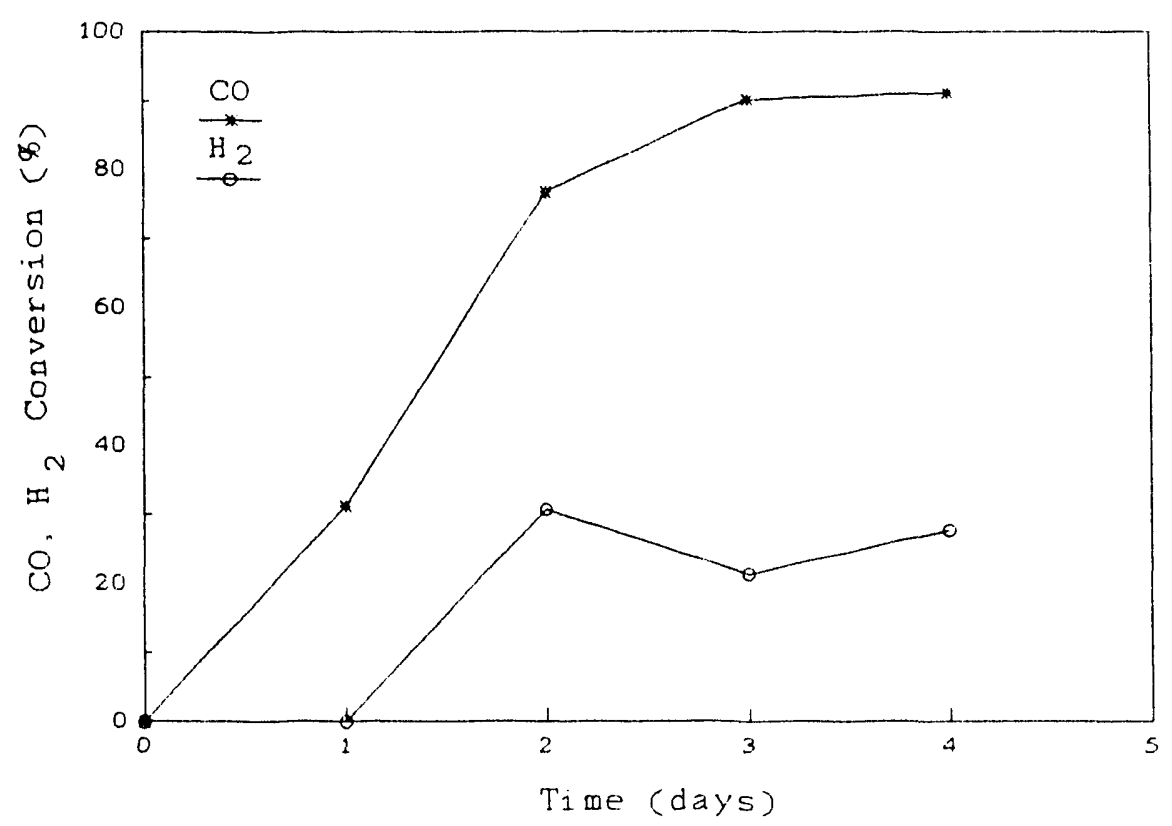

Figure 3. $\mathrm{CO}$ and $\mathrm{H}_{2}$ Consumption by Isolate ERI2 in the CSTR

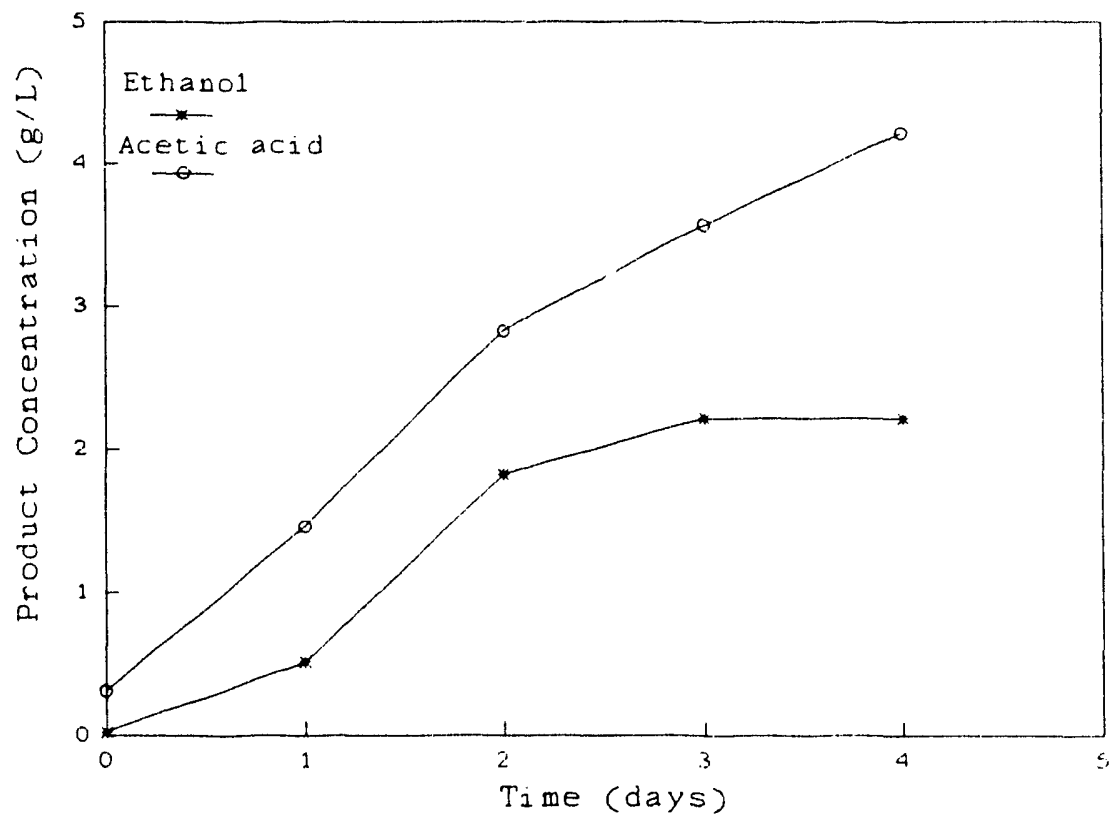

Figure 4. Ethanol and Acetic Acid Production by Isolate ERI2 in the CSTR

The performance of isolate ERI8 was also monitored with time in the CSTR (see Figures 5 and 6). As is shown in Figure 6, the $\mathrm{CO}$ conversion reached 90 percent and the $\mathrm{H}_{2}$ conversion 
reached 10 percent after 5 days of operation. The corresponding product concentrations were 0.5 $\mathrm{g} / \mathrm{L}$ ethanol and $4.0 \mathrm{~g} / \mathrm{L}$ acetate. It is hoped that this culture will improve toward ethanol production in the CSTR, especially in light of the encouraging results obtained earlier in batch culture.

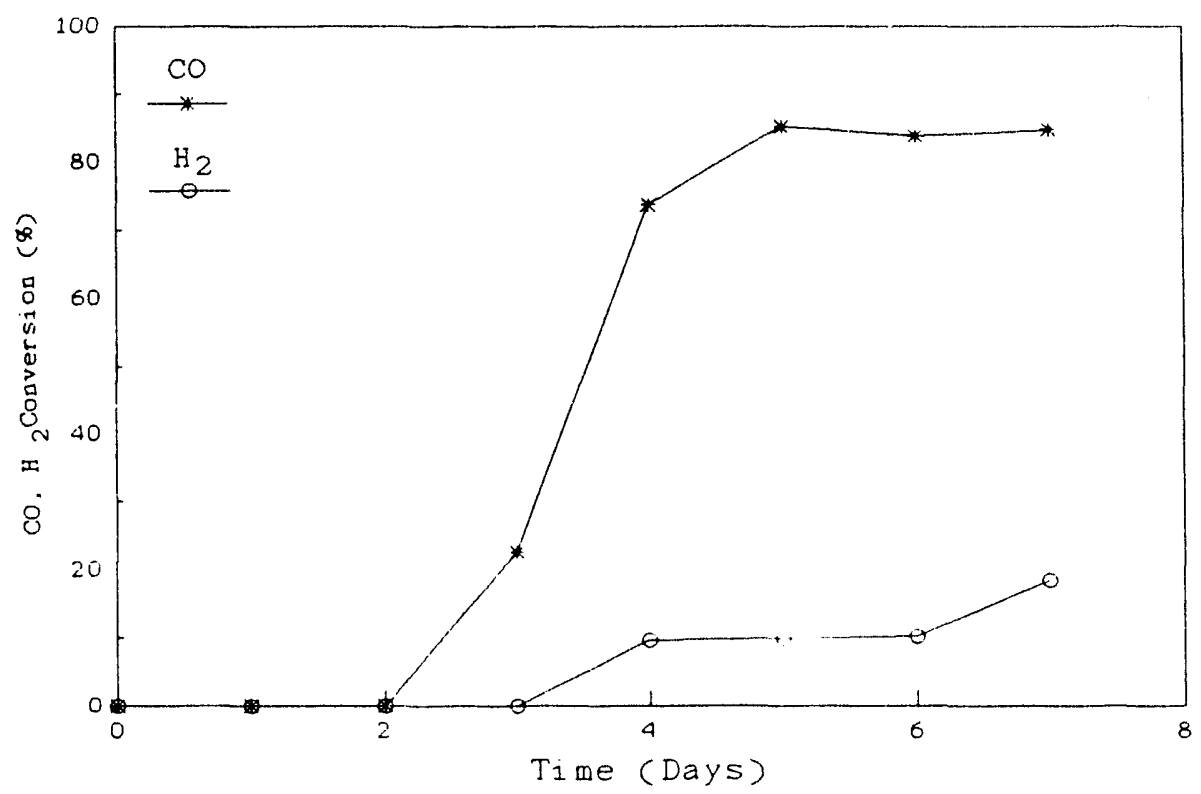

Figure 5. $\mathrm{CO}$ and $\mathrm{H}_{2}$ Consumption by Isolate ERI8 in the CSTR

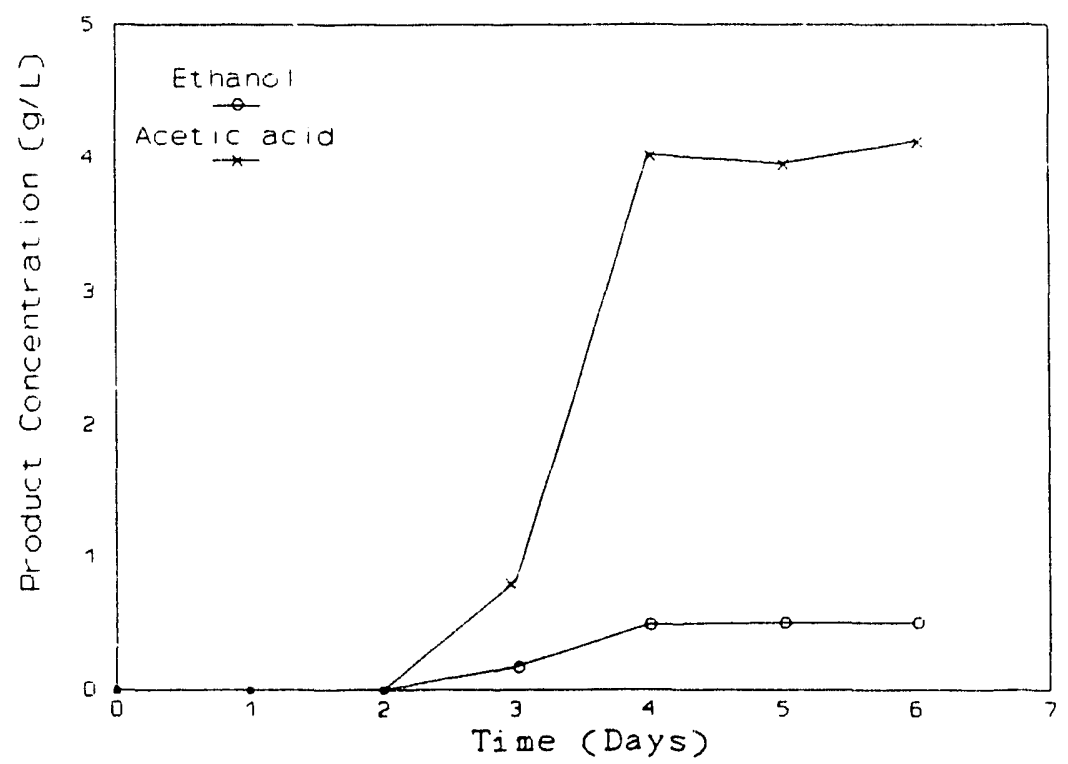

Figure 6. Ethanol and Acetic Acid Production from Synthesis Gas by Isolate ERI8 in the CSTR 
Task 3. Bench Scale Operation

Ethanol recovery data are being collected from the literature in order to develop the best system for the bench-scale unit. Options being considered are extraction, gas stripping, perstraction and pervaporation. Information from the literature search will be presented later. 

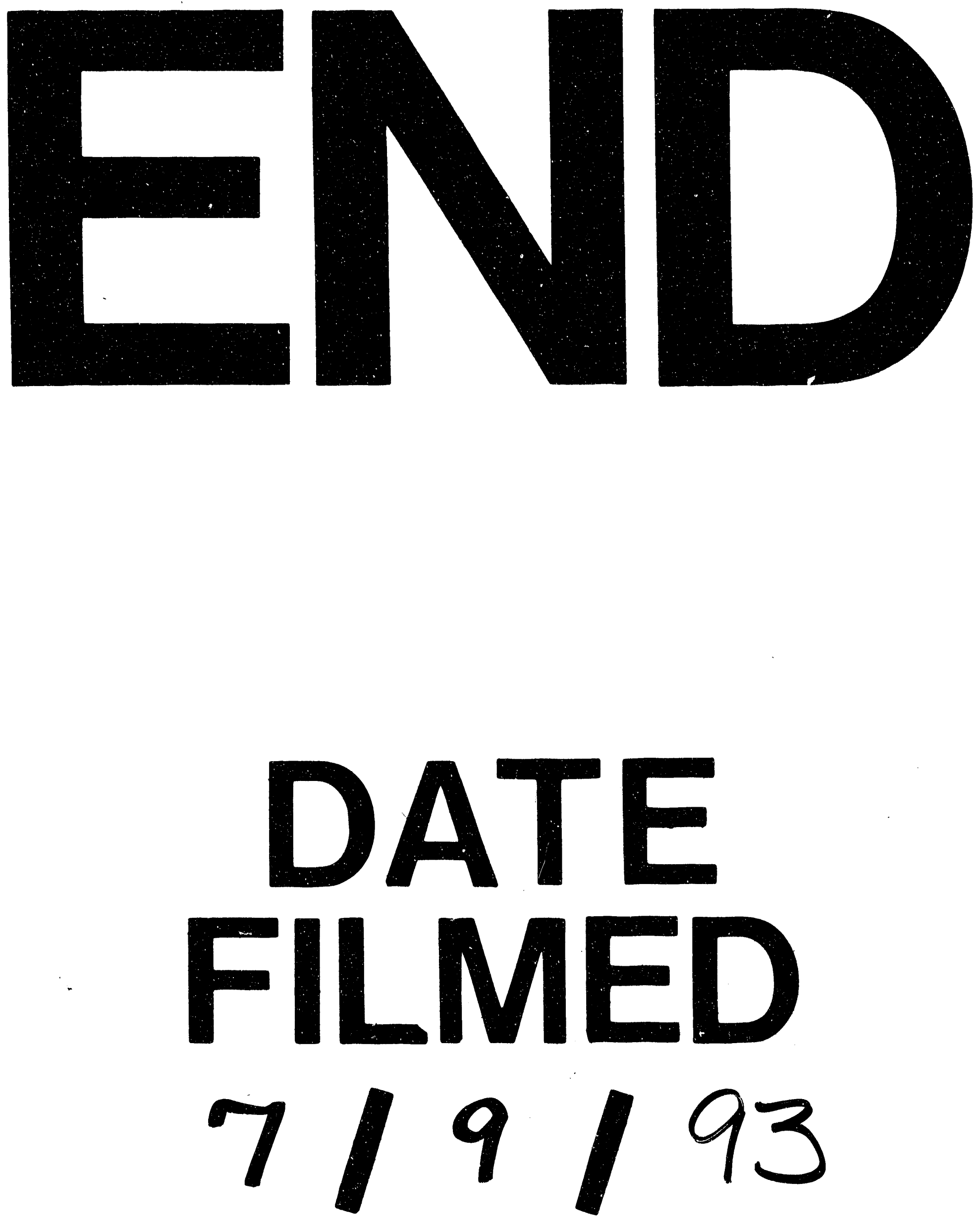

1 
